\title{
CERTAIN DENSE EMBEDDINGS OF REGULAR SEMIGROUPS
}

\author{
BY \\ MARIO PETRICH
}

\begin{abstract}
In a previous paper, the author has introduced a number of homomorphisms of an arbitrary semigroup into the translational hull of certain Rees matrix semigroups or orthogonal sums thereof. For regular semigroups, it is proved here that all of these homomorphisms have the property that the image is a densely embedded subsemigroup, i.e., is a densely embedded ideal of its idealizer, and that the corresponding Rees matrix semigroups are regular. Several of these homomorphisms are $1-1$, in each case they furnish a different dense embedding of an arbitrary regular semigroup into the translational hull of a regular Rees matrix semigroup or orthogonal sums thereof. A new representation for regular semigroups is introduced.
\end{abstract}

1. Introduction and summary. We have constructed in [7] a number of homomorphisms from an arbitrary or regular semigroup $S$ into the translational hull of a regular Rees matrix semigroup $T$, where $T$ is either the trace of a regular $\mathscr{D}$-class $D$ of $S$ or certain other regular Rees matrix semigroups associated either with $D$ or with $S$. Some of these homomorphisms (or their sums) are embeddings of $S$ into the translational hull (or their direct product) and, for certain classes of semigroups, some are closely related to irreducible representations by matrices over a field, transitive representations by partial transformations, etc.

This paper is essentially a supplement to [7]; for the sake of economy, we will not repeat any definitions, notation, or results that can be found in that paper. We prove here that for a regular semigroup $S$, letting $\varphi: S \rightarrow B$ stand for any of the homomorphisms of $S$ constructed in [7], $S \varphi$ is a densely embedded ideal of its idealizer $i_{B}(S \varphi)$ in $B$. For the case when $\varphi$ is $1-1, i_{B}(S \varphi)$ then furnishes a (natural) isomorphic copy of the translational hull $\Omega(S)$ of $S$. In $\S 2$ we prove this for $\chi_{D}: S \rightarrow \Omega\left(T_{D}\right)$ where $T_{D}$ is the trace of the $\mathscr{D}$-class $D$, and for the sum $\chi$ of all $\chi_{D}$. We perform a similar analysis in $\S 3$ for $\xi_{D}: S \rightarrow \Omega\left(F_{D}\right)$ where $F_{D}$ is the fragment of $D$, the sum $\xi$ of all $\xi_{D}$, and for $\xi \theta \delta: S \rightarrow \Omega(F)$ where $F$ is the fragment of $S$. In $\S 4$ we construct a homomorphism $\varepsilon$ of an arbitrary semigroup, which has as special cases $\eta$ and $\delta_{\sigma}$ of [7], and for a regular semigroup prove that $\varepsilon$ is an into isomorphism with the property stated above.

We now recall a minimum of needed definitions. If $S$ is an ideal of a semigroup

Received by the editors November 7, 1969 and, in revised form, February 5, 1970.

AMS 1969 subject classifications. Primary 2092.

Key words and phrases. Densely embedded ideal, idealizer, densely embedded subsemigroup, dense embedding, translational hull, regular semigroup, regular Rees matrix semigroup, trace of a $\mathscr{D}$-class, fragment of a $\mathscr{D}$-class, contour of a semigroup.

Copyright (C) 1971, American Mathematical Society 
$V$, then $V$ is an extension of $S ; V$ is a dense extension of $S$ if the equality congruence on $V$ is the only congruence on $V$ whose restriction to $S$ is the equality on $S$; if $V$ is a maximal (under inclusion) dense extension of $S$, then $S$ is a densely embedded ideal of $V$. If $A$ is a subsemigroup of a semigroup $B$, then the idealizer of $A$ in $B$ is given by $i_{B}(A)=\{b \in B \mid b a, a b \in A$ for all $a \in A\}$; if $A$ is a densely embedded ideal of $i_{B}(A)$, then $A$ is a densely embedded subsemigroup of $B$. If $\varphi$ is an isomorphism of $S$ into $B$ and $S \varphi$ is a densely embedded subsemigroup of $B$, we say that $\varphi$ is a dense embedding of $S$ into $B$ and also that $S$ can be densely embedded into $B$.

THEOREM. A weakly reductive semigroup $S$ is a densely embedded ideal of an extension $V$ if and only if the canonical homomorphism $\tau: V \rightarrow \Omega(S)$, defined by $\tau: v \rightarrow\left(\lambda_{\left.v\right|_{s}}, \rho_{\left.v\right|_{s}}\right)$ where $\lambda_{v}$ and $\rho_{v}$ are inner translations of $V$ induced by $v$, is an isomorphism of $V$ onto $\Omega(S)$.

(See $[2, \S 1]$ and $[4, \S 3]$.)

This theorem is crucial for most of the proofs in this paper. In fact, for a regular semigroup $S$ and a certain homomorphism $\varphi: S \rightarrow B$, in order to show that $S \varphi$ is a densely embedded subsemigroup of $B$, viz., that $S \varphi$ is a densely embedded ideal of $i_{B}(S \varphi)$, we prove that the canonical homomorphism $\tau: i_{B}(S \varphi) \rightarrow \Omega(S \varphi)$ is $1-1$ and onto. We will apply this idea and the above theorem without further mention.

Densely embedded ideals and subsemigroups play an important role in the study of various semigroups of (partial) transformations on a set, endomorphisms of a vector space, binary relations, several other "concrete" semigroups, and other algebraic structures (see [3]). A precedent in the theory of abstract semigroups which is the nearest analogue of some of the results in this paper is [8, 6.2].

2. Trace. The results in this section supplement those in [7, §3] and heavily depend upon them. We start with the trace of a $\mathscr{D}$-class of a regular semigroup.

THEOREM 1. For any $\mathscr{D}$-class $D$ of a regular semigroup $S, S \chi_{D}$ is a densely embedded subsemigroup of $\Omega\left(T_{D}\right)$.

Proof. We will show that the canonical homomorphism

$$
\tau_{D}: i_{\Omega\left(T_{D}\right)}\left(S \chi_{D}\right) \rightarrow \Omega\left(S \chi_{D}\right)
$$

is $1-1$ and onto.

1. To prove that $\tau_{D}$ is $1-1$, we suppose that $\omega, \omega^{\prime} \in i_{\Omega\left(T_{D}\right)}\left(S \chi_{D}\right)$ have the property $\omega \tau_{D}=\omega^{\prime} \tau_{D}$. Then in $\Omega\left(T_{D}\right)$ we have

$$
\omega\left(s \chi_{D}\right)=\omega^{\prime}\left(s \chi_{D}\right), \quad\left(s \chi_{D}\right) \omega=\left(s \chi_{D}\right) \omega^{\prime} \quad(s \in S)
$$

or, writing $\omega=(\lambda, \rho), \omega^{\prime}=\left(\lambda^{\prime}, \rho^{\prime}\right), s \chi_{D}=\left(\lambda^{s}, \rho^{s}\right)$, it follows that

$$
\lambda \lambda^{s}=\lambda^{\prime} \lambda^{s}, \quad \rho^{s} \rho=\rho^{s} \rho^{\prime} \quad(s \in S) .
$$

Now letting $a \lambda=(\alpha, \varphi)$ (recall that $\lambda(1 ; i, \mu)=(\varphi i ; \alpha i, \mu)$ if $\lambda(1 ; i, \mu) \neq 0$, and $a 0=0)$ 
and $a \lambda^{\prime}=\left(\alpha^{\prime}, \varphi^{\prime}\right)$ as in (4) of [6], using (6) of [6] and the definition of $\lambda^{s}$ in [7, §3], we obtain

$$
\lambda \lambda^{s}(a ; i, \mu)=\lambda\left(r_{j}^{\prime} s r_{i} a ; j, \mu\right)=\left((\varphi j) r_{j}^{\prime} s r_{i} a ; \alpha j, \mu\right)
$$

if $s H_{i 1}=H_{j 1}$ and $j \in d \alpha$, and 0 otherwise, and analogously

$$
\lambda^{\prime} \lambda^{s}(a ; i, \mu)=\left(\left(\varphi^{\prime} j\right) r_{j}^{\prime} s r_{i} a ; \alpha^{\prime} j, \mu\right)
$$

if $s H_{i 1}=H_{j 1}$ and $j \in d \alpha^{\prime}$, and 0 otherwise. Since $S$ is regular, for every pair $i, j$ of indices in $I$ an $s \in S$ can be found for which $s H_{i 1}=H_{j 1}$. Since by (1) we have $\lambda \lambda^{s}=\lambda^{\prime} \lambda^{s}$, it then follows that $j \in d \alpha \Leftrightarrow j \in d \alpha^{\prime}$, so that $\boldsymbol{d} \alpha=\boldsymbol{d} \alpha^{\prime}$, and hence also $\alpha j=\alpha^{\prime} j$ and $\varphi j=\varphi^{\prime} j$ for all $j \in d \alpha$. Consequently $\alpha=\alpha^{\prime}$ and $\varphi=\varphi^{\prime}$ which implies that $\lambda=\lambda^{\prime}$ since $a$ is $1-1$. Similarly the second equation in (1) implies that $\rho=\rho^{\prime}$, and hence $\omega=\omega^{\prime}$. Therefore $\tau_{D}$ is $1-1$.

2. To prove that $\tau_{D}$ is onto, we let $\left(\lambda^{\prime}, \rho^{\prime}\right) \in \Omega\left(S \chi_{D}\right)$ and must find $(\lambda, \rho) \in$ $i_{\Omega\left(T_{D}\right)}\left(S \chi_{D}\right) \subseteq \Omega\left(T_{D}\right)$ such that

$$
\begin{array}{ll}
\lambda^{\prime}\left(\lambda^{s}, \rho^{s}\right)=(\lambda, \rho)\left(\lambda^{s}, \rho^{s}\right)=\left(\lambda \lambda^{s}, \rho \rho^{s}\right) & (s \in S), \\
\left(\lambda^{s}, \rho^{s}\right) \rho^{\prime}=\left(\lambda^{s}, \rho^{s}\right)(\lambda, \rho)=\left(\lambda^{s} \lambda, \rho^{s} \rho\right) & (s \in S) .
\end{array}
$$

For every $s \in S$, write $\pi^{s}=s \chi_{D}=\left(\lambda^{s}, \rho^{s}\right)$. Let $C$ be any subset of $S$ intersecting each congruence class of ker $\chi_{D}$ exactly once, and for every $s \in S$, let $\bar{s}, \hat{s} \in C$ be defined by

$$
\lambda^{\prime} \pi^{s}=\pi^{s}, \quad \pi^{s} \rho^{\prime}=\pi^{s} .
$$

Next define

$$
\begin{aligned}
d \alpha & =\left\{j \in I \mid s H_{j 1}=H_{j 1}, \bar{s} H_{j 1}=H_{k 1} \text { for some } s \in S, k \in I\right\}, \\
\alpha j & =k, \quad \varphi j=\left(r_{k}^{\prime} \bar{s} r_{j}\right)\left(r_{j}^{\prime} s r_{j}\right)^{-1} \text { if } s H_{j 1}=H_{j 1}, \bar{s} H_{j 1}=H_{k 1}, \\
d \beta & =\left\{\nu \in \Lambda \mid H_{1 v} t=H_{1 v}, H_{1 v} \hat{t}=H_{1 \theta} \text { for some } t \in S, \theta \in \Lambda\right\}, \\
\nu \beta & =\theta, \quad \nu \psi=\left(q_{v} t q_{v}^{\prime}\right)^{-1}\left(q_{v} \hat{t} q_{\theta}^{\prime}\right) \text { if } H_{1 v} t=H_{1 v}, H_{1 v} \hat{t}=H_{1 \theta} .
\end{aligned}
$$

We will show that $(\lambda, \rho)$, where $\boldsymbol{a} \lambda=(\alpha, \varphi)$ and $\rho \boldsymbol{b}=(\psi, \beta)$, has the desired properties. Recall that $(s, t) \in \operatorname{ker} \chi_{D}$ if and only if for any $d \in D$,

$$
d s \mathscr{R} d \text { or } d t \mathscr{R} d \Rightarrow d s=d t \text { and } s d \mathscr{L} d \text { or } t d \mathscr{L} d \Rightarrow s d=t d .
$$

3. To show that $\alpha$ and $\varphi$ are single valued, we suppose that $s H_{j 1}=H_{j 1}$, $\bar{s} H_{j 1}=H_{k 1}$ and $t H_{j 1}=H_{j 1}, \bar{t} H_{j 1}=H_{n 1}$. Then $s r_{j} \mathscr{R} t r_{j}$ and hence $s r_{j}=t r_{j} u, t r_{j}=s r_{j} v$ for some $u, v \in S$ so that

$$
\begin{aligned}
\pi^{s r_{j}} & =\pi^{s} \pi^{r_{j}}=\left(\lambda^{\prime} \pi^{s}\right) \pi^{r_{j}}=\lambda^{\prime}\left(\pi^{s} \pi^{r_{j}}\right)=\lambda^{\prime} \pi^{s r_{j}} \\
& =\lambda^{\prime} \pi^{t r_{j} u}=\lambda^{\prime}\left(\pi^{t} \pi^{r_{j} u}\right)=\left(\lambda^{\prime} \pi^{t}\right) \pi^{r_{j} u}=\pi^{t} \pi^{r_{j} u}=\pi^{t_{r_{j}} u} .
\end{aligned}
$$

Since $\bar{s} r_{j} \in H_{k 1}$, we have $\bar{s} r_{j} \mathscr{L} e$ so that $\bar{s} r_{j}=\bar{s} r_{j} e$. On the other hand, (4) implies that $\left(\bar{s} r_{j}, \bar{t} r_{j} u\right) \in \operatorname{ker} \chi_{D}$ which together with $\bar{s} r_{j} \mathscr{L} e$ implies that $\left(\bar{s} r_{j}\right) e=\left(\bar{t} r_{j} u\right) e$. Consequently $\bar{s} r_{j}=\bar{t} r_{j}(u e) ;$ similarly $\bar{t} r_{j}=\bar{s} r_{j}(v e)$ and hence $\bar{s} r_{j} \mathscr{R} \bar{t} r_{j}$ which implies 
that $k=n$ and hence that $\alpha$ is single valued. Further, we may suppose that $u \in H_{11}$ since $H_{j 1} H_{11}=H_{j 1}$ and obtain

$$
\left(r_{k}^{\prime} \bar{s} r_{j}\right)\left(r_{j}^{\prime} s r_{j}\right)^{-1}=\left(r_{k}^{\prime} \bar{t} r_{j} u e\right)\left(r_{j}^{\prime} t r_{j} u\right)^{-1}=\left(r_{k}^{\prime} \bar{t} r_{j}\right) u u^{-1}\left(r_{k}^{\prime} t r_{j}\right)^{-1}=\left(r_{k}^{\prime} \bar{t} r_{j}\right)\left(r_{k}^{\prime} t r_{j}\right)^{-1}
$$

which implies that $\varphi$ is single valued. An analogous argument shows that both $\psi$ and $\beta$ are single valued.

4. To establish that $\lambda$ and $\rho$ are linked, by [6, Theorem 3] we must show:

$$
\begin{aligned}
& s H_{j 1}=H_{j 1}, \bar{s} H_{j 1}=H_{k 1} \text { for some } s \in S \text { and } q_{v} r_{k} \in H_{11} \\
& \Leftrightarrow H_{1 v} t=H_{1 v}, H_{1 v} \hat{t}=H_{1 \theta} \text { for some } t \in S \text { and } q_{\theta} r_{j} \in H_{11} \\
& \Rightarrow\left(q_{v} r_{k}\right)\left(r_{k}^{\prime} \bar{s} r_{j}\right)\left(r_{j}^{\prime} s r_{j}\right)^{-1}=\left(q_{v} t q_{v}^{\prime}\right)^{-1}\left(q_{v} \hat{t} q_{\theta}^{\prime}\right)\left(q_{\theta} r_{j}\right) .
\end{aligned}
$$

Suppose that $s H_{j 1}=H_{j 1}, \bar{s} H_{j 1}=H_{k 1}, q_{v} r_{k} \in H_{11}$. Let $t \in S$ be such that $H_{1 v} t=H_{1 v}$; then

$$
\pi^{t s}=\pi^{t} \pi^{s}=\pi^{t}\left(\lambda^{\prime} \pi^{s}\right)=\left(\pi^{t} \rho^{\prime}\right) \pi^{s}=\pi^{\hat{t}} \pi^{s}=\pi^{\hat{t} s}
$$

whence $\pi^{q_{v}{ }^{t} s r_{j}}=\pi^{q_{v} t_{s} r_{j}}$ where $\left(q_{v} t\right)\left(\bar{s} r_{j}\right) \in H_{1 v} H_{k 1}=H_{11}$ since $q_{v} r_{k} \in H_{11}$. Hence $e \mathscr{L}\left(q_{v} t \bar{s} r_{j}\right) e$ which together with $\left(q_{v} t \bar{s} r_{j}, q_{v} \hat{t} s r_{j}\right) \in \operatorname{ker} \chi_{D}$ implies that $\left(q_{v} t \bar{s} r_{j}\right) e$ $=\left(q_{v} \hat{t} s r_{j}\right) e$. But then $q_{v} t \bar{s} r_{j}=q_{v} \hat{t} s r_{j}$ since $r_{j} \mathscr{L} e$ implies $r_{j} e=r_{j}$. Consequently $q_{v} \hat{t} s r_{j} \in H_{11}$ which implies that $H_{1 v} \hat{t} s r_{j}=H_{11}$. It follows that $H_{1 v} \hat{t}=H_{1 \theta}$ for some $\theta \in \Lambda$ by [1, Lemma 3.15 (ii)]. Further, $q_{\theta} \in H_{1 v} \hat{t}$ and $r_{j} \in s H_{j 1}$ so that $H_{1 v} \hat{t} s r_{j}=H_{11}$ implies that $q_{\theta} r_{j} \in H_{11}$. We have proved: $H_{1 v} t=H_{1 v}, H_{1 v} \hat{t}=H_{1 \theta}$ for some $t \in S$ and $q_{\theta} r_{j} \in H_{11}$; the converse implication in (5) is proved analogously.

Suppose next that the two equivalent statements in (5) hold. We have seen that $q_{v} t \bar{s} r_{j}=q_{v} \hat{s} s r_{j}$. Since $q_{v} t \in H_{1 v}$, we have $q_{v} t \mathscr{L} f_{v}$ so that $q_{v} t=q_{v} t f_{v}$ and $f_{v}=q_{v}^{\prime} q_{v}$ and thus $q_{v} t=q_{v} t q_{v}^{\prime} q_{v}$; similarly $s r_{j}=r_{j} r_{j}^{\prime} s r_{j}$. Consequently

$$
\left(q_{v} t q_{v}^{\prime}\right)\left(q_{v} \bar{s} r_{j}\right)=\left(q_{v} t\right)\left(\bar{s} r_{j}\right)=\left(q_{v} t r_{j}\right)\left(r_{j}^{\prime} s r_{j}\right),
$$

and since $r_{j}^{\prime} s r_{j}, q_{v} t q_{v}^{\prime} \in H_{11}$, it follows that $\left(q_{v} \bar{s} r_{j}\right)\left(r_{j}^{\prime} s r_{j}\right)^{-1}=\left(q_{v} t q_{v}^{\prime}\right)^{-1}\left(q_{v} t r_{j}\right)$. Further $\bar{s} r_{j} \in H_{k 1}$ implies that $\bar{s} r_{j} \mathscr{R} e_{k}$ which together with $e_{k}=r_{k} r_{k}^{\prime}$ implies $\bar{s} r_{j}=e_{k} \bar{s} r_{j}$, $=r_{k} r_{k}^{\prime} \bar{s} r_{j} ;$ similarly $q_{v} \hat{t}=q_{v} \hat{t} q_{\theta}^{\prime} q_{\theta}$. Therefore

$$
\left(q_{v} r_{k}\right)\left(r_{k}^{\prime} \bar{s} r_{j}\right)\left(r_{j}^{\prime} s r_{j}\right)^{-1}=\left(q_{v} t q_{v}^{\prime}\right)^{-1}\left(q_{v} \hat{t} q_{\theta}^{\prime}\right)\left(q_{\theta} r_{j}\right)
$$

which completes the proof of (5) and shows that $(\lambda, \rho) \in \Omega\left(T_{D}\right)$.

5. We prove next the first part of (2), viz., $\lambda \lambda^{s}=\lambda^{s}$. On the one hand

$$
\begin{aligned}
\lambda \lambda^{s}(a ; i, \mu) & =\lambda\left(\left(r_{j}^{\prime} s r_{i}\right) a ; j, \mu\right)=\left((\varphi j)\left(r_{j}^{\prime} s r_{i}\right) a ; \alpha j, \mu\right) \\
& =\left(\left(r_{k}^{\prime} \bar{t} r_{j}\right)\left(r_{j}^{\prime} t r_{j}\right)^{-1}\left(r_{j}^{\prime} s r_{i}\right) a ; k, \mu\right)
\end{aligned}
$$

if $s H_{i 1}=H_{j 1}, t H_{j 1}=H_{j 1}, \bar{t} H_{j 1}=H_{k 1}$, and 0 otherwise, and on the other hand $\lambda^{s}(a ; i, \mu)=\left(\left(r_{l}^{\prime} s r_{i}\right) a ; l, \mu\right)$ if $\bar{s} H_{i 1}=H_{l 1}$, and 0 otherwise. Hence we must prove

$$
\begin{aligned}
& s H_{i 1}=H_{j 1} \text { and } t H_{j 1}=H_{j 1}, \bar{t} H_{j 1}=H_{k 1} \text { for some } t \in S \\
& \Leftrightarrow \bar{s} H_{i 1}=H_{k 1} \Rightarrow\left(r_{k}^{\prime} \bar{t} r_{j}\right)\left(r_{j}^{\prime} t r_{j}\right)^{-1}\left(r_{j}^{\prime} s r_{i}\right)=r_{k}^{\prime} \bar{s} r_{i} .
\end{aligned}
$$


Suppose that $s H_{i 1}=H_{j 1}, t H_{j 1}=H_{j 1}, i H_{j 1}=H_{k 1}$. Then $t r_{i}, r_{j} \in s H_{j 1}$ and hence $t r_{j}=s r_{i} v$ for some $v \in H_{11}$ since $H_{j 1} H_{11}=H_{j 1}$. Consequently $\pi^{t r_{j}}=\pi^{s r_{i} v}$ so that $\pi^{t_{r_{j}}}=\pi^{s r_{i} v}$ and thus $\left(\bar{t}_{r_{j}}, \bar{s} r_{i} v\right) \in \operatorname{ker} \chi_{D}$. Since $\bar{t} r_{j} \mathscr{L} e$, we conclude as before that $\bar{t} r_{j}=\bar{t} r_{j} e=\bar{s} r_{i} v e=\bar{s} r_{i} v$. Here $r_{i} v \in H_{i 1} H_{11}=H_{i 1}$ and $\bar{t} r_{j} \in H_{k 1}$ and hence $\bar{s} H_{i 1}=H_{k 1}$ as desired.

Conversely, suppose that $\bar{s} H_{i 1}=H_{k 1}$. Then $\lambda^{s}(a ; i, \mu) \neq 0$, and since $S$ is regular, $s=s d s$ for some $d \in S$. Hence

$$
0 \neq \lambda^{s}(a ; i, \mu)=\pi^{s}(a ; i, \mu)=\left(\lambda^{\prime} \pi^{s}\right)(a ; i, \mu)=\left(\lambda^{\prime} \pi^{s}\right) \pi^{d}\left[\lambda^{s}(a ; i, \mu)\right],
$$

which implies that $\lambda^{s}(a ; i, \mu) \neq 0$ so that $s H_{i 1}=H_{j 1}$ for some $j \in I$. There exists $v \in S$ such that $v H_{j 1}=H_{i 1}$. It follows easily that $\pi^{\overline{s v} r_{j}}=\pi^{s v r_{j}}$, further $\bar{s} v r_{j} \in \bar{s} v H_{j 1}$ $=\bar{s} H_{i 1}=H_{k 1}$, and as before we conclude that $\overline{s v} r_{j}=\bar{s} v r_{j}$ so that $\overline{s v} H_{j 1}=\bar{s} v H_{j 1}$ $=\bar{s} H_{i 1}=H_{k 1}$. Therefore $t=s v$ is the desired element which proves the converse in (6).

Suppose next that the equivalent statements in (6) are satisfied. Hence $s H_{i 1}=t H_{j 1}$ so that $s r_{i}=t u$ for some $u \in H_{j 1}$. As before we conclude that $\bar{s} r_{i}=\bar{t} u$ and that $u=e_{j} u=r_{j} r_{j}^{\prime} u$ which yields

$$
\begin{aligned}
\left(r_{k}^{\prime} \bar{t} r_{j}\right)\left(r_{j}^{\prime} t r_{j}\right)^{-1}\left(r_{j}^{\prime} s r_{i}\right) & =\left(r_{k}^{\prime} \bar{t} r_{j}\right)\left(r_{j}^{\prime} t r_{j}\right)^{-1}\left(r_{j}^{\prime} t u\right) \\
& =\left(r_{k}^{\prime} \bar{t} r_{j}\right)\left(r_{j}^{\prime} t r_{j}\right)^{-1}\left(r_{j}^{\prime} t r_{j}\right) r_{j}^{\prime} u \\
& =\left(r_{k}^{\prime} \bar{t} r_{j}\right) r_{j}^{\prime} u=r_{k}^{\prime} \bar{t} u=r_{k}^{\prime} \bar{s}_{i} .
\end{aligned}
$$

Therefore $\lambda \lambda^{s}=\lambda^{s}$.

6. We now prove the second part of (2), viz., $\rho \rho^{s}=\rho^{s}$. On the one hand

$$
(a ; i, \mu) \rho \rho^{s}=\left(a\left(q_{\mu} t q_{\mu}^{\prime}\right)^{-1}\left(q_{\mu} \hat{t} q_{\theta}^{\prime}\right) ; i, \theta\right) \rho^{s}=\left(a\left(q_{\mu} t q_{\mu}^{\prime}\right)^{-1}\left(q_{\mu} \hat{t} q_{\theta}^{\prime}\right)\left(q_{\theta} s q_{\gamma}^{\prime}\right) ; i, \gamma\right)
$$

if $H_{1 \mu} t=H_{1 \mu}, H_{1 \mu} \hat{t}=H_{1 \theta}, H_{1 \theta} s=H_{1 \gamma}$, and 0 otherwise, and on the other hand $(a ; i, \mu) \rho^{s}=\left(a\left(q_{\mu} \bar{s} q_{\delta}^{\prime}\right) ; i, \delta\right)$ if $H_{1 \mu} \bar{s}=H_{1 \delta}$, and 0 otherwise. Hence we must prove

$$
\begin{aligned}
& H_{1 \mu} t=H_{1 \mu}, H_{1 \mu} \hat{t}=H_{1 \theta} \text { for some } t \in S \text { and } H_{1 \theta} s=H_{1 \gamma} \\
& \Leftrightarrow H_{1 \mu} \bar{s}=H_{1 \gamma} \Rightarrow\left(q_{\mu} t q_{\mu}^{\prime}\right)^{-1}\left(q_{\mu} \hat{t} q_{\theta}^{\prime}\right)\left(q_{\theta} s q_{\gamma}^{\prime}\right)=q_{\mu} \bar{s} q_{\gamma}^{\prime} .
\end{aligned}
$$

Suppose that $H_{1 \mu} t=H_{1 \mu}, H_{1 \mu} \hat{t}=H_{1 \theta}, H_{1 \theta} s=H_{1 \gamma}$. We have seen above that $\pi^{t s}=\pi^{t} s$ so that $(t \bar{s}, \hat{t} s) \in \operatorname{ker} \chi_{D}$. Since $q_{\mu} \hat{t} s \in H_{1 \mu} \hat{t} s=H_{10} s=H_{1 \gamma}$ and $\left(q_{\mu} t \bar{s}, q_{\mu} \hat{t} s\right)$ $\in$ ker $\chi_{D}$, similarly as before we conclude that $q_{\mu} t \bar{s}=q_{\mu} \hat{t} s$. Consequently

$$
H_{1 \mu} \bar{s}=H_{1 \mu} t \bar{s}=H_{1 \mu} \hat{t} s=H_{10} s=H_{1 \gamma} .
$$

Conversely, suppose that $H_{1 \mu} \bar{s}=H_{1 \gamma}$. There exists $t \in S$ such that $H_{1 \mu} t=H_{1 \mu}$. Then $H_{1 \mu} t \bar{s}=H_{1 \gamma}$ and similarly as before we conclude that $q_{\mu} \hat{i} s=q_{\mu} t \bar{s}$ and thus $H_{1 \mu} \hat{t} s=H_{1 \gamma}$. Hence by [1, Lemma 3.15 (ii)] we have that $H_{1 \mu} \hat{t}=H_{1 \theta}$ for some $\theta \in \Lambda$ and thus also $H_{10} s=H_{1 \gamma}$.

Now assume the validity of the two equivalent statements in (7). Since $q_{\mu} \hat{t} \in H_{1 \theta}$, we have $q_{\mu} \hat{t} \mathscr{L} f_{\theta}$ which together with $f_{\theta}=q_{\theta}^{\prime} q_{\theta}$ implies $q_{\mu} \hat{t}=q_{\mu} \hat{t} q_{\theta}^{\prime} q_{\theta}$; similarly 
$q_{\mu} t \in H_{1 \mu}$ implies $q_{\mu} t=q_{\mu} t q_{\mu}^{\prime} q_{\mu}$. We have seen above that $q_{\mu} \hat{t} s=q_{\mu} t \bar{s}$ so that $\left(q_{\mu} \hat{t} q_{\theta}^{\prime}\right)\left(q_{\theta} s q_{\gamma}^{\prime}\right)=\left(q_{\mu} t q_{\mu}^{\prime}\right)\left(q_{\mu} \bar{s} q_{\gamma}^{\prime}\right)$ where each expression in parentheses is in $H_{11}$. Thus

$$
\left(q_{\mu} t q_{\mu}^{\prime}\right)^{-1}\left(q_{\mu} \hat{t} q_{\theta}^{\prime}\right)\left(q_{\theta} s q_{\gamma}^{\prime}\right)=q_{\mu} \bar{s} q_{\gamma}^{\prime}
$$

which completes the proof of (7). Therefore $\rho \rho^{s}=\rho^{s}$.

7. In 5 and 6 above, we have established (2); formula (3) follows analogously. Therefore $(\lambda, \rho) \tau_{D}=\left(\lambda^{\prime}, \rho^{\prime}\right)$ which proves that $\eta$ maps $i_{\Omega\left(T_{D}\right)}\left(S \chi_{D}\right)$ onto $\Omega\left(S_{\chi_{D}}\right)$ and completes the proof of the theorem.

We now consider the sum $\chi$ of $\chi_{D}$ as $D$ ranges over the index set $\Delta$ of all $\mathscr{D}$-classes of a regular semigroup.

THEOREM 2. For $S$ any regular semigroup, $\chi$ is a dense embedding of $S$ into $\prod_{D \in \Delta} \Omega\left(T_{D}\right)$.

Proof. Let $\tau: i_{\prod_{D \in \Delta} \Omega\left(T_{D}\right)}\left(S_{\chi}\right) \rightarrow \Omega\left(S_{\chi}\right)$ be the canonical homomorphism, we must show that $\tau$ is $1-1$ and onto. Suppose that $\omega \tau=\omega^{\prime} \tau$. Then

$$
\omega(s \chi)=\omega^{\prime}(s \chi), \quad(s \chi) \omega=(s \chi) \omega^{\prime} \quad(s \in S),
$$

or, writing $\omega=\left(\omega_{D}\right)_{D \in \Delta}, \omega^{\prime}=\left(\omega_{D}^{\prime}\right)_{D \in \Delta}$ where $\omega_{D}, \omega_{D}^{\prime} \in i_{\Omega\left(T_{D}\right)}\left(S \chi_{D}\right)$ for every $D \in \Delta$, we have

$$
\omega_{D}\left(s \chi_{D}\right)=\omega_{D}^{\prime}\left(s \chi_{D}\right), \quad\left(s \chi_{D}\right) \omega_{D}=\left(s \chi_{D}\right) \omega_{D}^{\prime} \quad(s \in S, D \in \Delta) .
$$

For a fixed $D \in \Delta$, it follows by Theorem 1 that $\omega_{D}=\omega_{D}^{\prime}$ which then implies that $\omega=\omega^{\prime}$. Therefore $\tau$ is $1-1$.

To prove that $\tau$ is onto, we let $\left(\lambda^{\prime}, \rho^{\prime}\right) \in \Omega\left(S_{\chi}\right)$ and must find $\omega \in i_{\prod_{D \in \Delta} \Omega\left(T_{D}\right)}\left(S_{\chi}\right)$ such that

$$
\lambda^{\prime}(s \chi)=\omega(s \chi), \quad(s \chi) \rho^{\prime}=(s \chi) \omega \quad(s \in S),
$$

or, writing $\omega=\left(\left(\lambda_{D}, \rho_{D}\right)\right)_{D \in \Delta}, s \chi=\left(s \chi_{D}\right)_{D \in \Delta}=\left(\left(\lambda_{D}^{s}, \rho_{D}^{s}\right)\right)_{D \in \Delta}$,

$$
\lambda^{\prime}(s \chi)=\left(\left(\lambda_{D} \lambda_{D}^{s}, \rho_{D} \rho_{D}^{s}\right)\right)_{D \in \Delta}, \quad(s \chi) \rho^{\prime}=\left(\left(\lambda_{D}^{s} \lambda_{D}, \rho_{D}^{s} \rho_{D}\right)\right)_{D \in \Delta} \quad(s \in S) .
$$

Since $\chi$ is $1-1$, for every $s \in S$, we may define $\bar{s}$ and $\hat{s}$ by

$$
\lambda^{\prime}(s \chi)=\bar{s} \chi, \quad(s \chi) \rho^{\prime}=\hat{s} \rho .
$$

Formulae (9) written componentwise then become

$$
\lambda_{D}^{s}=\lambda_{D} \lambda_{D}^{s}, \quad \rho_{D}^{s}=\rho_{D} \rho_{D}^{s}, \quad \lambda_{D}^{s}=\lambda_{D}^{s} \lambda_{D}, \quad \rho_{D}^{s}=\rho_{D}^{s} \rho_{D} \quad(s \in S, D \in \Delta) .
$$

For a fixed $D \in \Delta$, we define $(\alpha, \varphi)$ and $(\psi, \beta)$ as in the proof of Theorem 1 and let $a \lambda_{D}=(\alpha, \varphi), \rho_{D} b=(\psi, \beta)$. Following the same steps as in the part of the proof of Theorem 1 after the definitions of $(\alpha, \varphi),(\psi, \beta)$, and, in fact, going through the same proof except for the simplifications arising from the fact that $\chi$ is $1-1$, one shows that $\left(\lambda_{D}, \rho_{D}\right) \in \Omega\left(T_{D}\right)$ and that all the formulae in (10) hold. Consequently (9) also holds and thus $\omega=\left(\left(\lambda_{D}, \rho_{D}\right)\right)_{D \in \Delta}$ satisfies the two equations in (8), which proves that $\omega \tau=\left(\lambda^{\prime}, \rho^{\prime}\right)$. Therefore $\tau$ is onto.

The next corollary sharpens [7, Theorem 2]. 
COROLlARY. Every regular semigroup can be densely embedded both into the direct product of the translational hulls of the traces of its different $\mathscr{D}$-classes and into the translational hull of its trace.

Proof. This follows from Theorem 2 and the proof of [7, Theorem 2].

3. Fragment. The results here supplement those of $[7, \S 4]$ and depend upon them.

TheOREM 3. For any $\mathscr{D}$-class $D$ of a regular semigroup $S, S \xi_{D}$ is a densely embedded subsemigroup of $\Omega\left(F_{D}\right)$.

Proof. We must show that the canonical homomorphism $\tau_{D}: i_{\Omega\left(F_{D}\right)}\left(S \xi_{D}\right) \rightarrow$ $\Omega\left(S \xi_{D}\right)$ is $1-1$ and onto.

Suppose that $\omega, \omega^{\prime} \in i_{\Omega\left(F_{D}\right)}\left(S \xi_{D}\right)$ have the property $\omega \tau_{D}=\omega^{\prime} \tau_{D}$. Then letting $\omega=(\lambda, \rho), \omega^{\prime}=\left(\lambda^{\prime}, \rho^{\prime}\right)$ and $a \lambda=\alpha, a \lambda^{\prime}=\alpha^{\prime}$ as in (4) of [6], we get for any $l \in L_{1}$ and $u$ an inverse of $l$,

$$
\begin{aligned}
\lambda \lambda^{l}(1 ; u l, r) & =\lambda(1 ; l, r)=(1 ; \alpha l, r) \text { if } l \in d \alpha, \text { and } 0 \text { otherwise, } \\
\lambda^{\prime} \lambda^{l}(1 ; u l, r) & =\lambda^{\prime}(1 ; l, r)=\left(1 ; \alpha^{\prime} l, r\right) \text { if } l \in d \alpha^{\prime}, \text { and } 0 \text { otherwise. }
\end{aligned}
$$

Since $\omega\left(l \xi_{D}\right)=\omega^{\prime}\left(l \xi_{D}\right)$, it follows that $\lambda \lambda^{l}=\lambda^{\prime} \lambda^{l}$ for all $l \in L_{1}$, which by (11) implies that $\alpha=\alpha^{\prime}$ whence $\lambda=\lambda^{\prime}$. A similar argument proves that also $\rho=\rho^{\prime}$ and thus $\omega=\omega^{\prime}$. Consequently $\tau_{D}$ is $1-1$.

To show that $\tau_{D}$ is onto, let $\left(\lambda^{\prime}, \rho^{\prime}\right) \in \Omega\left(S \xi_{D}\right)$. Let $C$ be any subset of $S$ intersecting each congruence class of ker $\xi_{D}$ exactly once, for every $s \in S$ write $\pi^{s}=s \xi_{D}$ $=\left(\lambda^{s}, \rho^{s}\right)$, and let $\bar{s}, \hat{s} \in C$ be defined by $\lambda^{\prime} \pi^{s}=\pi^{s}, \pi^{s} \rho^{\prime}=\pi^{s}$. Next define

$$
\begin{aligned}
d \alpha & =\left\{l \in L_{1} \mid s l=l, \bar{s} l \in L_{1} \text { for some } s \in S\right\}, \\
\alpha l & =\bar{s} l \text { if } s l=l \in L_{1}, \bar{s} l \in L_{1}, \\
d \beta & =\left\{r \in R_{1} \mid r t=r, r \hat{t} \in R_{1} \text { for some } t \in S\right\}, \\
r \beta & =r \hat{t} \text { if } r t=r \in R_{1}, r \hat{t} \in R_{1} .
\end{aligned}
$$

We will show that $(\lambda, \rho) \tau_{D}=\left(\lambda^{\prime}, \rho^{\prime}\right)$ where $a \lambda=\alpha, \rho b=\beta$. First note that $(s, t) \in \operatorname{ker} \xi_{D}$ if and only if for any $l \in L_{1}: s l \in L_{1}$ or $t l \in L_{1} \Rightarrow s l=t l$ and for any $r \in R_{1}: r s \in R_{1}$ or $r t \in R_{1} \Rightarrow r s=r t$.

Suppose that $l=s l=t l$ with $l, \bar{s} l, i t l \in L_{1}$. Then $\pi^{s l}=\pi^{t l}$ so that $\pi^{s l}=\pi^{t l}$ and hence $(\bar{s} l, \bar{t} l) \in \operatorname{ker} \xi_{D}$. Since $l, \bar{s} l, \bar{t} l, e \in L_{1}$, it follows that $\bar{s} l=(\bar{s} l) e=(\bar{t} l) e=\bar{t} l$, which proves that $\alpha$ is single valued; similarly $\beta$ is single valued.

To show that $\lambda$ and $\rho$ are linked, by [6, Theorem 3] we must prove that for any $l \in L_{1}, r \in R_{1}$,

$$
\begin{aligned}
& s l=l, \bar{s} l \in L_{1}, r \bar{s} l=e \text { for some } s \in S \\
& \Leftrightarrow r t=r, r \hat{t} \in R_{1}, r \hat{t} l=e \text { for some } t \in S .
\end{aligned}
$$


Suppose that $s l=l, \bar{s} l \in L_{1}, r \bar{s} l=e$, with $l \in L_{1}, r \in R_{1}$. Then $\pi^{i l}=\pi^{t s l}=\pi^{r s l}=\pi^{e}$ so that $(\hat{r} l, e) \in \operatorname{ker} \xi_{D}$. Since $l, e \in L_{1}$, we obtain $\hat{r} l=\hat{r} l e=e e=e$. Let $u$ be an inverse of $r$, and let $t=u r$. Then $r t=r$ and hence $(r \hat{t}, \hat{r}) \in \operatorname{ker} \xi_{D}$. Since $l, \hat{r} l \in L_{1}$, it follows that $r \hat{t} l=\hat{r} l$ and hence $r \hat{t} l=e$. Further, $r, e \in R_{1}$ implies that $r=e r$ so $r \hat{t}=e(r \hat{t})$ which together with $e=(r \hat{t}) l$ implies that $r \hat{t} \mathscr{R} e$ and thus $r \hat{t} \in R_{1}$. We have proved that $r t=r, r \hat{t} \in R_{1}, r \hat{t} l=e$; the converse implication in (12) is established analogously. Consequently $(\lambda, \rho) \in \Omega\left(F_{D}\right)$.

For any $s \in S$, we have

$$
\begin{aligned}
& \lambda \lambda^{s}(1 ; l, r)=(1 ; \bar{t} s l, r) \text { if } s l, \bar{t} s l \in L_{1}, t s l=s l \text { for some } t \in S, \text { and } 0 \text { otherwise, } \\
& \lambda^{s}(1 ; l, r)=(1 ; \bar{s} l, r) \text { if } \bar{s} l \in L_{1} \text {, and } 0 \text { otherwise. }
\end{aligned}
$$

If $s l, \bar{t} s l \in L_{1}$ and $t s l=s l$, then $(\bar{t} s l, \bar{s} l) \in \operatorname{ker} \xi_{D}$ and thus $\bar{s} l=\bar{s} l e=\bar{t} s l e=\bar{t} s l \in L_{1}$. Conversely, suppose that $\bar{s} l \in L_{1}$, let $u$ be an inverse of $s$, and $t=s u$. Then $t s l=s l$ and hence $(\bar{t} s l, \bar{s} l) \in \operatorname{ker} \xi_{D}$, and similarly as above, we conclude that $\bar{t} s l=\bar{s} l \in L_{1}$. It follows that $\bar{s} l \mathscr{L} l$ which yields $s l \mathscr{L} l$, that is, $s l \in L_{1}$. Consequently $\lambda \lambda^{s}=\lambda^{s}$.

Further

$$
\begin{aligned}
(1 ; l, r) \rho \rho^{s} & =(1 ; l, r \hat{t} s) \text { if } r \hat{t}, r \hat{t} s \in R_{1}, r t=r \text { for some } t \in S, \text { and } 0 \text { otherwise, } \\
(1 ; l, r) \rho^{s} & =(1 ; l, r \bar{s}) \text { if } r \bar{s} \in R_{1}, \text { and } 0 \text { otherwise. }
\end{aligned}
$$

An argument similar to the preceding one shows that $\rho \rho^{s}=\rho^{s}$. A dual proof establishes $\lambda^{s} \lambda=\lambda^{3}$ and $\rho^{s} \rho=\rho^{\hat{s}}$ which proves that $(\lambda, \rho) \tau_{D}=\left(\lambda^{\prime}, \rho^{\prime}\right)$.

We consider next the sum $\xi$ of all $\xi_{D}$.

THEOREM 4. For any regular semigroup $S, \xi$ is a dense embedding of $S$ into $\prod_{D \in \Delta} \Omega\left(F_{D}\right)$.

Proof. The argument here is entirely analogous to that in the proof of Theorem 2 if we substitute $\chi$ by $\xi$ and use Theorem 3 instead of Theorem 1. For the definitions of $\theta$ and $\delta$, see the proofs of [7, Lemma 1] and [7, Lemma 3], respectively.

THEOREM 5. For any regular semigroup $S, \xi \theta \delta$ is a dense embedding of $S$ into $\Omega(F)$.

Proof. First note that by [7, Lemma 1] and Theorem 4, we have that $K=S \xi \theta$ is a densely embedded ideal of $i_{\Omega(N)}(K)$ where $N=\sum_{D \in \Delta} \oplus F_{D}$. Hence $K \delta$ is a densely embedded ideal of

$$
\left(i_{\Omega(N)}(K)\right) \delta=i_{\Omega(F)}(K \delta) \cap(\Omega(N)) \delta .
$$

Thus to prove the theorem, it suffices to show that $i_{\Omega(F)}(K \delta)$ is a dense extension of $K \delta$. Recall that for any $s \in S$,

$$
s \xi \theta \delta=\left(\lambda_{D}^{s}, \rho_{D}^{s}\right)_{D \in \Delta} \theta \delta=\left(\lambda^{s}, \rho^{s}\right) \delta=\left(\phi^{s}, \psi^{s}\right)
$$


where $\lambda^{s} x=\lambda_{D}^{s} x$ and $x \rho^{s}=x \rho_{D}^{s}$ if $x \in F_{D}, \lambda^{s} 0=0 \rho^{s}=0$, and

$$
F=\mathscr{M}^{\circ}\left(1 ; \bigcup_{D \in \Delta} L_{1}^{D}, \bigcup_{D \in \Delta} R_{1}^{D} ; P\right), \quad P=\left(p_{r l}\right)
$$

with $p_{r l}=1$ if $r \in R_{1}^{D}, l \in L_{1}^{D}, r l=e_{D}$ for some $D \in \Delta$, and 0 otherwise, and for any $(1 ; l, r) \in F, \varphi^{s}(1 ; l, r)=\left(1 ; \alpha_{D}^{s} l, r\right)$ if $l \in L_{1}^{D}, a\left(\left.\lambda^{s}\right|_{F_{D}}\right)=\alpha_{D}^{s}, l \in d \alpha_{D}^{s}$, and 0 otherwise, and a corresponding expression for $(1 ; l, r) \psi^{s}, \varphi^{s} 0=0 \psi^{s}=0$.

Now suppose that $(\lambda, \rho),\left(\lambda^{\prime}, \rho^{\prime}\right) \in i_{\Omega(F)}(K \delta)$ have the property

$$
(\lambda, \rho)\left(\varphi^{s}, \psi^{s}\right)=\left(\lambda^{\prime}, \rho^{\prime}\right)\left(\varphi^{s}, \psi^{s}\right), \quad\left(\varphi^{s}, \psi^{s}\right)(\lambda, \rho)=\left(\varphi^{s}, \psi^{s}\right)\left(\lambda^{\prime}, \rho^{\prime}\right) \quad(s \in S),
$$

so that

$$
\lambda \varphi^{s}=\lambda^{\prime} \varphi^{s}, \quad \rho \psi^{s}=\rho^{\prime} \psi^{s}, \quad \varphi^{s} \lambda=\varphi^{s} \lambda^{\prime}, \quad \psi^{s} \rho=\psi^{s} \rho^{s} \quad(s \in S) .
$$

Let $l \in L_{1}^{D}$ and $u$ be an inverse of $l$. Then $u l \in L_{1}^{D}$ and letting $a \lambda=\alpha, a \lambda^{\prime}=\alpha^{\prime}$, we obtain

$$
\begin{aligned}
\lambda \varphi^{l}(1 ; u l, r) & =(1 ; \alpha l, r) \text { if } l \in d \alpha, \text { and } 0 \text { otherwise, } \\
\lambda^{\prime} \varphi^{l}(1 ; u l, r) & =\left(1 ; \alpha^{\prime} l, r\right) \text { if } l \in d \alpha^{\prime}, \text { and } 0 \text { otherwise. }
\end{aligned}
$$

It follows that $\alpha=\alpha^{\prime}$ and hence $\lambda=\lambda^{\prime}$; similarly $\rho=\rho^{\prime}$ which proves that $(\lambda, \rho)$ $=\left(\lambda^{\prime}, \rho^{\prime}\right)$. Therefore $i_{\Omega(F)}(K \delta)$ is a dense extension of $K \delta$. Since $i_{\Omega(F)}(K \delta) \cap(\Omega(N)) \delta$ is a maximal dense extension of $K \delta$, it follows that $i_{\Omega(F)}(K \delta)=\left(i_{\Omega(N)}(K)\right) \delta$. Therefore $K \delta$ is a densely embedded ideal of $i_{\Omega(F)}(K \delta)$ and thus a densely embedded subsemigroup of $\Omega(F)$.

The next corollary sharpens [7, Theorem 5].

COROLlaRY. Every regular semigroup can be densely embedded into

(i) the direct product of the translational hulls of the fragments of its different $\mathscr{D}$-classes,

(ii) the translational hull of the orthogonal sum of the fragments of its different D-classes,

(iii) the translational hull of its fragment.

4. Another representation of a regular semigroup. In [7, $\$ 5$ and 6] we have introduced representations of a regular semigroup $S$ using the $\mathscr{L}$ and $\mathscr{R}$ equivalences on $S$ (into the contour of $S$ ) and using a semilattice congruence on $S$. We now introduce a "common ancestor" to both of these representations by making use of conditions $\left(T_{r}\right)$ and $\left(T_{l}\right)$ stated in [5, Chapter II, $\left.\S 1\right]$ and refer the reader to this source for further applications of these conditions to representations of regular semigroups. In the discussion that follows and in Proposition 1, the semigroup $S$ need not be regular.

Let $\gamma$ and $\delta$ be binary relations on $S$ satisfying the conditions:

$$
\begin{array}{ll}
a \delta a b c \Leftrightarrow a \delta a b, a b \delta a b c & (a, b, c \in S), \\
a \gamma c b a \Leftrightarrow a \gamma b a, b a \gamma c b a & (a, b, c \in S) .
\end{array}
$$


Let $A=\mathscr{M}^{\circ}(1 ; S, S ; P)$ where $P=\left(p_{a b}\right)$ is an $S \times S$ matrix with $p_{a b}=1$ if $a \delta a b$ and $b \gamma a b$, and 0 otherwise. For every $s \in S$, define the functions $\lambda^{s}$ and $\rho^{s}$ on $A$ by

$$
\begin{aligned}
\lambda^{s}(1 ; x, y) & =(1 ; s x, y) \text { if } x y s x, \text { and } 0 \text { otherwise, } \\
(1 ; x, y) \rho^{s} & =(1 ; x, y s) \text { if } y \delta y s, \text { and } 0 \text { otherwise, } \\
\lambda^{s} 0 & =0 \rho^{s}=0,
\end{aligned}
$$

and let $\varepsilon: s \rightarrow\left(\lambda^{s}, \rho^{s}\right)(s \in S)$.

Proposition 1. For any semigroup $S, \varepsilon$ is a homomorphism of $S$ into $\Omega(A)$ and

$$
\operatorname{ker}\left(\varepsilon \pi_{\mathrm{P}}\right)=\{(s, t) \mid \text { if } x \in S \text { and either } x \delta x s \text { or } x \delta x t \text {, then } x s=x t\} \text {. }
$$

Proof. The proof that $\lambda^{s}$ is a left translation and that $\lambda^{s} \lambda^{t}=\lambda^{s t}$ is almost the same as in [7, Theorem 3]; the case of right translations is dual. An obvious modification of the corresponding part of the proof of [7, Theorem 6] shows that $\lambda^{s}$ and $\rho^{s}$ are linked and that $\operatorname{ker}\left(\varepsilon \pi_{\mathrm{P}}\right)$ is the one given in the statement of the proposition.

It is clear that [7, Theorem 6] corresponds to the case $\gamma=\mathscr{L}$ and $\delta=\mathscr{R}$, and [7, Theorem 7] to the case $\gamma=\delta=\sigma$. These theorems are further sharpened in the following more general result.

THEOREM 6. Let $S$ be a regular semigroup, let $\gamma$ and $\delta$ be reflexive binary relations on $S$ satisfying conditions (13) and (14), respectively, and let $A$ and $\varepsilon$ be as defined above relative to $\gamma, \delta$. Then $A$ is a regular Rees matrix semigroup and $\varepsilon$ is a dense embedding of $S$ into $\Omega(A)$.

Proof. Let $a \in S$ and let $b$ be an inverse of $a$. Then $a \delta a b a$ so that $a \delta a b$; also $b \gamma b a b$ so that $b \gamma a b$. Consequently $p_{a b}=1$ and similarly $p_{b a}=1$; hence the sandwich matrix $P$ of $A$ is regular. Suppose next that $(s, t) \in \operatorname{ker} \varepsilon$. Then for any inverses $s^{\prime}$ and $t^{\prime}$ of $s$ and $t$, respectively, using the expression for $\operatorname{ker}\left(\varepsilon \pi_{\mathrm{P}}\right)$ in Proposition 1 and its dual for ker $\left(\varepsilon \pi_{\Lambda}\right)$, we obtain: $s^{\prime} \delta s^{\prime} s s^{\prime}$ implies $s^{\prime} \delta s^{\prime} s$ and hence $s^{\prime} t=s^{\prime} s$ and similarly $t^{\prime} s=t^{\prime} t$; dually $t s^{\prime}=s s^{\prime}, s t^{\prime}=s s^{\prime}$. Consequently

$$
s=s s^{\prime} s=t s^{\prime} s=t t^{\prime} t s^{\prime} s=t t^{\prime} s s^{\prime} s=t t^{\prime} s=t t^{\prime} t=t .
$$

We will show again that the canonical homomorphism $\tau: i_{\Omega(A)}(S \varepsilon) \rightarrow \Omega(S \varepsilon)$ is 1-1 and onto. The proof that $\tau$ is 1-1 is almost identical to the proof in Theorem 3 that $\tau_{D}$ is $1-1$. To show that $\tau$ is onto, we let $\left(\lambda^{\prime}, \rho^{\prime}\right) \in \Omega(S \varepsilon)$. Since $\varepsilon$ is $1-1$, for every $s \in S$, we may define $\bar{s}$ and $\hat{s}$ by $\lambda^{\prime}(s \varepsilon)=\bar{s} \varepsilon$ and $(s \varepsilon) \rho^{\prime}=\hat{s} \varepsilon$. Now define

$$
\begin{array}{ll}
d \alpha=\{s x \in S \mid x \gamma \bar{s} x\}, & \alpha(s x)=\bar{s} x \text { if } x \gamma \bar{s} x, \\
d \beta=\{y t \in S \mid y \delta y \hat{t}\}, & (y t) \beta=y \hat{t} \text { if } y \delta y \hat{t} .
\end{array}
$$

We show next that $(\lambda, \rho) \tau=\left(\lambda^{\prime}, \rho^{\prime}\right)$ where $a \lambda=\alpha, \rho b=\beta$. Since $\varepsilon$ is $1-1$, it follows easily that $\overline{s x}=\bar{s} x$. Hence if $s x=t y$, then $\bar{s} x=\overline{s x}=\overline{t y}=\bar{t} y$ and $\alpha$ is single valued; 
similarly $\beta$ is single valued. To show that $\lambda$ and $\rho$ are linked, we let $(1 ; x, y)$, $(1 ; z, w) \in A$. There exist $e, f \in S$ such that $y=y e, z=f z$, and hence

$$
(1 ; x, y)[\lambda(1 ; z, w)]=(1 ; x, y)[\lambda(1 ; f z, w)]=(1 ; x, w)
$$

if $z \gamma \bar{f} z, y \delta y \bar{f} z, \bar{f} z \gamma y \bar{f}$, and 0 otherwise; similarly

$$
[(1 ; x, y) \rho](1 ; z, w)=[(1 ; x, y e) \rho](1 ; z, w)=(1 ; x, w)
$$

if $y \delta y \hat{e}, y \hat{e} \delta y \hat{e} z, z \gamma y \hat{e} z$, and 0 otherwise. Taking into account that $y \bar{f} z=y e \bar{f} z$ $=y \hat{e} f z=y \hat{e} z$ and the conditions on $\gamma$ and $\delta$, it follows immediately that each of the sets of conditions $z \gamma \bar{f} z, y \delta y \bar{f} z, \bar{f} z \gamma y \bar{f} z$ and $y \delta$ ye, yê $\delta$ yê, $z \gamma$ yêz is equivalent to $y \delta$ yêz, $z$ y yêz. Therefore $(\lambda, \rho) \in \Omega(A)$.

Further,

$$
\begin{aligned}
\lambda \lambda^{s}(1 ; x, y) & =(1 ; \bar{s} x, y) \text { if } x \gamma s x, x \gamma \bar{s} x, \text { and } 0 \text { otherwise, } \\
\lambda^{s}(1 ; x, y) & =(1 ; \bar{s} x, y) \text { if } x \gamma \bar{s} x, \text { and } 0 \text { otherwise. }
\end{aligned}
$$

Let $x \gamma \bar{s} x$ and let $e$ be a left identity of $\bar{s}$. Then $x \gamma \bar{s} x$ and $\bar{s} x \gamma e \bar{s} x$ imply $x \gamma e \bar{s} x$. But then $x \gamma \hat{e} s x$ which in turn implies that $x \gamma s x$. Consequently, it follows from (15) that $\lambda \lambda^{s}=\lambda^{s}$.

Let $y \in S$ and let $f$ be a right identity of $y$; then

$$
\begin{aligned}
(1 ; x, y) \rho \rho^{s} & =(1 ; x, y \hat{s}) \text { if } y \delta y \hat{f}, y \hat{f} \delta y \hat{f} s, \text { and } 0 \text { otherwise, } \\
(1 ; x, y) \rho^{s} & =(1 ; x, y \bar{s}) \text { if } y \delta y \bar{s}, \text { and } 0 \text { otherwise. }
\end{aligned}
$$

Noting that $y \hat{f} s=y f \bar{s}=y \bar{s},(16)$ immediately yields that $\rho \rho^{s}=\rho^{s}$.

A similar proof shows that $\lambda^{s} \lambda=\lambda^{s}$ and $\rho^{s} \rho=\rho^{s}$, which then implies that $(\lambda, \rho) \tau=\left(\lambda^{\prime}, \rho^{\prime}\right)$ and completes the proof.

COROLlaRY. Every regular semigroup can be densely embedded into the translational hull of its contour.

\section{REFERENCES}

1. A. H. Clifford and G. B. Preston, The algebraic theory of semigroups. Vol. I, Math. Surveys, no. 7, Amer. Math. Soc., Providence, R. I., 1961. MR 24 \#A2627.

2. L. M. Gluskin, Ideals of semigroups, Mat. Sb. 55 (97) (1961), 421-448. (Russian) MR 25 \#3106.

3. - On dense embeddings, Mat. Sb. 61 (103) (1963), 175-206. (Russian) MR 27 \#2570.

4. P. A. Grillet and M. Petrich, Ideal extensions of semigroups, Pacific J. Math. 26 (1968), 493-508. MR 38 \#5967.

5. G. Lallement, Demi-groupes réguliers, Ann. Mat. Pura Appl. (4) 77 (1967), 47-129. MR 37 \#1505.

6. M. Petrich, The translational hull of a completely 0-simple semigroup, Glasgow Math. J. 9 (1968), 1-11. MR 37 \#5313.

7. - Representations of semigroups and the translational hull of a regular Rees matrix semigroup, Trans. Amer. Math. Soc. 143 (1969), 303-318.

8. I. S. Ponizovskili, Semigroups with given ideal chains, Mat. Sb. 61 (103) (1963), 377-388. (Russian) MR 27 \#5850.

Pennsylvania State University, University Park, Pennsylvania 16802 